\title{
Maternal Perinatal Mental Health and Multiple Births: Implications for Practice
}

\author{
Jane Fisher' and Andrew Stocky ${ }^{2}$ \\ 'Key Centre for Women's Health in Society, School of Population Health, University of Melbourne,Victoria, Australia \\ ${ }^{2}$ The Melbourne Clinic Consulting Suites, Richmond,Victoria, Australia
}

\begin{abstract}
W omen's mental health can be compromised during reproductive life, but to date there has been relatively little specific investigation of the links between multiple births and perinatal psychiatric illness. There has been more comprehensive examination of some of the psychological sequelae of multiple gestations and births, but many of the studies have small samples and are descriptive in nature. Most of the literature is drawn from investigations of the psychological aspects of multiple births following assisted conception. Current conceptualizations of the determinants of maternal perinatal mental health, with particular reference to multiple gestations and births are discussed and implications for clinical practice suggested.

Overall there is evidence that women with multiple gestation and multiple births may be at elevated risk for pregnancy anxiety, postpartum depression and complicated grief reactions. Much less is currently known about the associations between multiple birth and either maternity blues or postpartum psychosis. The relationships between personal or family psychiatric history, past experience of childhood abuse, intimate partner intimidation and psychological adjustment to multiple births are not known. The interactions between multiple births, operative delivery, prematurity, neonatal illness and separation of mother and infant as contributing factors to maternal postpartum mental health are not known. There is very limited evidence about the psychological functioning of fathers of multiple infants. Routine antenatal, intrapartum and postnatal health care for women with multiple infants needs to take into account the additional psychological demands they face.
\end{abstract}

The birth of an infant demands dramatic adaptations of women. In becoming a mother a woman has to relinquish her autonomy, personal liberty, occupational identity, capacity to generate an income and social and leisure activities in service of infant care. The adaptation to new required roles, major responsibilities, generational shift, multiplication of the unpaid workload, and, for some, harm to bodily integrity through unexpected adverse reproductive events places great demands on individual psychological resources and existing relationships. All of these are greater and the outcomes less certain in women who conceive and give birth to multiple infants.

\section{Psychology and Psychiatry of Pregnancy, Childbirth and the Postpartum Year}

Psychological disequilibrium is normal during life transitions and in adaptation to change, and there is continuing theoretical consideration of the extent to which perinatal psychological disorder should be regarded as part of a normal process. There is now, however, substantial evidence that women's mental health can be compromised in reproductive life and some experience psychiatric illness. Debate continues about whether psychiatric illnesses occurring in pregnancy or after childbirth are clinically distinct from those observed at other phases of the life cycle, and of the relative etiological contributions of biological and psychosocial factors. There has been relatively little systematic investigation of the distinct perinatal psychological sequelae of spontaneously conceived multiple pregnancies, but there has been more focus on multiple pregnancies following assisted conception.

\section{Multiple Gestation Pregnancy}

Maternal anxiety about infant health and wellbeing is observable in all pregnancies, but intensified with multiple gestations. The diagnosis of a multiple gestation is usually unexpected, and can be greeted with intense anxiety and fear of being overwhelmed, in particular among those with no family history of multiple births. Presence of the partner and empathic professional communication of the diagnosis have a beneficial impact on initial adjustment (Bryan \& Denton, 2001; Evans et al., 2001).

Multiple pregnancy can be idealised after prolonged infertility and parents may underestimate the hazards. Couples in this situation can seek a multiple birth actively in order to create an instant family (Gleicher et al., 1995; Goldfarb et al., 1996; Leiblum et al., 1990). Although parents may have significant fears about fetal wellbeing and their own capacity to adapt to the work of caring for multiple infants, they can feel prohibited in expressing this ambivalence because they consented to the transfer of multiple embryos (Klock, 2001). Multiple gestations are usually diagnosed very early after assisted conception and parents may not think about the possibility of loss of one fetus. Grief in those who have a single baby after having lost another fetus during pregnancy is intense, but can be unrecognised both by health professionals and friends

Address for correspondence: Jane Fisher, Key Centre for Women's Health in Society, School of Population Health, University of Melbourne, VIC 3010, Australia.Email: jrwf@unimelb.edu.au 
(Kollantai \& Fleischer, 2001; Swanson et al., 2002). The coincidence of bereavement and pregnancy presents a psychological paradox, which is not easily resolved and may lead to delayed or disturbed grief reactions or disrupt the growth of secure mother-infant attachment (Bryan \& Denton, 2001; Fisher, 2002; Pector \& Smith-Levitin, 2002). Very little is known about the long-term psychological consequences of planned multifetal pregnancy reduction in multiple pregnancies, although the maintenance of secrets harms family relationships (Bryan, 2002). Qualitative investigations report complicated grief reactions in which multiple disenfranchised losses are experienced (Collopy, 2002). The potential impact on the living infant remains unknown, but the possibility that a "survivor syndrome" characterised by guilt, perplexity and grief for a lost sibling has been suggested (Bryan \& Denton, 2001).

Historically, antenatal depression has been under-recognised, in part because some symptoms of depression (fatigue, appetite change and sleep disturbance) are normal in pregnancy. Recent evidence is that rates of depression are at least as high (10\%-15\%) among pregnant women as among those who have recently given birth (Evans et al., 2001). Antenatal depression predicts depression after childbirth. There are few comprehensive comparisons of rates of clinically significant anxiety and depressive symptomatology between women with single and multiple pregnancies, but Leonard (1998) in summarising the available literature reports little difference. The exception is among mothers with a multiple pregnancy and at least one older child, $30 \%$ of whom were found in one study to be depressed in the last trimester of pregnancy (Hay et al., 1990). Mothers in this circumstance worry about the potential disruption to older children if they are unable to provide normal care because of enforced bed rest in late pregnancy (Hay et al., $1990)$ and the impact of being displaced by two infants (Bryan \& Denton, 2001). Mothers pregnant with twins may have disproportionate feelings of unattractiveness associated with weight gain and fear rejection (Weigel et al., 2000). The quality of antenatal emotional attachment between a mother and her growing babies, and her overall adjustment, are positively associated with having a supportive confiding relationship with her partner (Colpin et al., 1998; Weigel et al., 2000)

\section{Childbirth}

Operative interventions in birth and prematurity, both more common among women with multiple gestations carry independent psychological costs (Barrett \& Ritchie, 2002). There is consistent evidence that instrumental intervention in vaginal birth and cesarean surgery are associated with elevated anxiety, disappointment, grief and dissatisfaction (Fisher et al., 1997). Although mode of delivery does not appear to make an independent contribution to postpartum depression when other risk factors are taken into account (Johnstone et al., 2001), cesarean surgery can induce posttraumatic stress reactions (Fisher et al., 1997). After cesarean childbirth the first encounter between mother and infant and the initiation of breastfeeding are disrupted with adverse effects on maternal confidence still measurable 8 months later (Rowe-Murray \& Fisher, 2002).
Premature birth, typically involves separation of the mother from her infant, sometimes for prolonged periods. Intense anxiety about infant health and development and about the separation is universal, but more severe among those whose infants are seriously unwell, and can persist for up to 3 years. During hospitalization in neonatal intensive care units mothers report uncertainty about whether the health professional or the parent has authority regarding the baby (Bryan \& Denton, 2001; Loo et al., 2003) and can be aided by a cooperative approach to decision-making that respects parental rights. Fear about infant health and viability can persist and lead to hyper vigilance about infant care and delayed formation of a secure affectionate emotional attachment. (Klock, 2001; Loo et al., 2003). Few of the existing studies examine the interacting emotional effects of operative birth, prematurity, neonatal hospitalisation and multiple births, but the established difficulties are further magnified if the infants are separated, for example by discharge of one infant ahead of another or by care in different hospitals (Bryan \& Denton, 2001). Maintaining a supply of breast milk and establishing breastfeeding are difficult and anxiety-arousing with premature and very low birthweight infants. Mothers of multiple infants can establish breastfeeding, but require sensitive and skilled care that takes account of maternal anxiety with a flexible, encouraging and non-judgmental approach (Colonna et al., 1997).

There is now a consistent view that psychological disturbance following childbirth can be conceptualised as fitting one of three distinct conditions of differing severity.

\section{Postpartum Blues or Mild Transient Mood Disturbance}

Maternity, third day or postpartum "blues" occur in up to $80 \%$ of parturient women in the days immediately following childbirth (Steiner, 1998). The syndrome is characterised by a range of symptoms, most commonly: a lability of mood between euphoria and misery, heightened sensitivity, tearfulness, often without associated sadness, restlessness, poor concentration, anxiety and irritability (Steiner, 1998). Disturbed sleep and feelings of unreality and detachment from the baby(ies) are also reported. The coincidence of the maternity blues with the major hormonal changes associated with parturition has led to investigations of a biological basis to the condition, but findings are generally inconsistent (Steiner, 1998). Similarly, there is no consistent evidence for the contribution of parity (Kendell et al., 1981), obstetric factors (Condon \& Watson, 1987), hospital or home as place of delivery (Kendell et al., 1981), or personal or family history of mood disorder (O'Hara et al., 1991) to incidence or severity of the condition. The distress peaks between days 3 and 5 postpartum and usually resolves spontaneously without specialist intervention. However, in some women a more persistent and severe depression develops. There is some evidence that intensity of blues symptomatology, including early self-reports of feeling depressed, having thoughts about death or being unable to stop crying predict later development of depression (O'Hara et al., 1991). There are no systematic investigations available of the impact of multiple births on the incidence of postpartum blues; however, this emotional lability is likely to amplify 
the psychological demands for a mother grappling with the realities of caring for more than one infant.

\section{Psychotic Illnesses}

A very small group of women (approximately 1-2 per 1000) develop the most severe psychiatric illness associated with childbirth: an acute functional psychosis within the first month postpartum. Relative lifetime risk and incidence are usually calculated in terms of psychiatric admission for treatment of psychotic illness after childbirth. It is highly elevated for the first 30 days of the postpartum period and remains elevated, but at a lower rate for the 2 years following childbirth (Pfuhlmann et al., 2002; Scottish Intercollegiate Guidelines Network, 2002). Clinical characteristics include acute onset, extreme affective variation, with mania and elation as well as sadness, thought disorder, delusions, hallucinations, disturbed behaviour and confusion (Pfuhlmann et al., 2002; Scottish Intercollegiate Guidelines Network, 2002). Postpartum psychoses are most accurately construed as episodes of cycloid affective illnesses and rates of schizophrenic psychotic episodes are not elevated postnatally (Pfuhlmann et al., 2002). Although treatment is similar, a divergence of views exists as to whether puerperal psychotic episodes occurring in an individual with an existing diagnosis of bipolar affective disorder should be understood to be the same as first episodes following childbirth (Pfuhlmann et al., 2002). Risk of recurrence after subsequent pregnancies is between 51\% and 69\% (Pfuhlmann et al., 2002). Postpartum psychosis has been associated with primiparity, personal or family history of affective psychosis, single marital status and perinatal death. The contribution of obstetric factors is not clear, but there is some evidence that cesarean delivery increases the risk of postpartum psychosis and of relapse after subsequent births. Future puerperal and non-puerperal episodes of psychosis are predicted most strongly by history of a previous psychotic episode and by marital difficulties (Pfuhlmann et al., 2002). As yet the relationship between multiple births and postpartum psychosis has not been investigated.

\section{Postpartum Depression}

It is well established that women experience Major Depression at between 1.6 and 2.6 times the lifetime rate reported for men and that this difference is most apparent in the life phase of caring for infants and young children (Epperson, 1999). Depression arising after childbirth has attracted very substantial research interest in the last four decades and there is now a copious literature devoted to the nature, prevalence, prediction, course and associations with risk and protective factors of postpartum depression in women. Most conceptualisations take a categorical approach in which individuals are classified as satisfying the criteria for clinical caseness, or are regarded as well. Others (Fisher et al., 2002) argue that adjustment processes including transient dysphoria or symptoms of depression are observable in most parturient women and that a continuum of emotional wellbeing or a broader spectrum of adjustment experiences may be a more accurate conceptualization. The relative contributions of biological and psychosocial etiological factors to postpartum depression are debated. Current summary and systematic reviews have concluded that although some women may be particularly psychologically vulnerable to hormonal change, as yet no direct link between hormones or other neurochemicals and postpartum depression has been demonstrated (Scottish Intercollegiate Guidelines Network, 2002). There is more substantial, consistent and robust evidence that a range of psychosocial factors contribute to postpartum depression. The reviews concur that postpartum depression is unlikely to be attributable to a single cause, but is probably governed by the interaction of a number of risk and protective factors (Scottish Intercollegiate Guidelines Network, 2002) and is dynamic (Evans et al., 2001). It is of serious public health concern because of the demonstrated adverse consequences it has on the development of maternal confidence and the cognitive, emotional and social development of these women's infants (Murray et al., 1999).

Postpartum depression is characterised by the persistent presence for at least 2 weeks of cognitive and affective symptoms including: low mood, guilt, despondency, self-deprecation, anhedonia, impaired concentration, irritability, elevated anxiety, rumination and social withdrawal. The somatic symptoms of sleep and appetite disturbance are also present, but are a feature of normal postpartum adjustment. There is consistent evidence from industrialised countries that $10-15 \%$ of parturient women will experience non-psychotic clinical depression in the year after giving birth, with a threefold increase in onset in the first 5 postpartum weeks (Epperson, 1999). Severe illnesses needing inpatient psychiatric treatment occur in $3-7 \%$ of parturient women (O'Hara et al., 1991).

There is consistent evidence that the emotional health of mothers of multiple infants is poorer and they are at higher risk of becoming depressed, anxious and clinically exhausted after childbirth than mothers of single infants. Hay and colleagues (1990) found that compared to mothers of 3-month-old single infants, $29.7 \%$ of mothers of twins of comparable age reported depression (five times higher rate) and $42 \%$ had high anxiety (three times higher rate). Even higher rates of depression have been reported among mothers of triplets. Robin et al. (1991) found that $40 \%$ were depressed 4 months postpartum and Garel and Blondel (1992) found that all reported significant distress and a quarter were being treated for depression 1-year postpartum. Two studies of the health and social circumstances of women admitted to Australian residential early parenting services for treatment of unsettled infant behaviour and maternal exhaustion, anxiety and depression have found an excess of mothers with multiple infants $(3.7 \%-5 \%)$ compared to the general population rate of multiplicity of $1.4 \%$ (Barnett et al., 1993; Fisher et al., 2002). In a longitudinal study of more than 13,000 mothers and children born in one month in 1970, Thorpe et al. (1991) found that independent of other explanatory factors, mothers of 5-yearold twins were three times more likely to be depressed than those with a single child.

A poor relationship with a partner is now regarded as a major predictor of depression after childbirth in women (Scottish Intercollegiate Guidelines Network, 2002). The flaws in this relationship have been variously conceptualised and reported as increased marital conflict; partner being 
less available after delivery; providing insufficient practical support or poor emotional support, poor adjustment or an unhappy relationship; low satisfaction; insufficient involvement in infant care or holding traditional rigid sex-role expectations (Scottish Intercollegiate Guidelines Network, 2002; Wilson et al., 1996). Relationship with partner appears to be significant both in the onset of depression and in the time taken to recovery of emotional wellbeing. Partners who are able to provide encouragement, reassurance, containment and unconditional affection may protect against depression (Fisher et al., 2002).

Wider theorising has suggested that depressed women are more likely to be irritable and socially withdrawn and therefore to be difficult for their partners to relate to, to be providing less care for their partners or to perceive their relationships as poor. There are also suggestions that men's mental health might be adversely affected by childbirth. Marks et al. (1992) postulate that men may feel excluded from the intimate relationship between mother and infant and themselves become depressed or anxious. There has been much less systematic examination of perinatal psychological functioning in men than in women. However, men are not at elevated risk of psychotic illness after the birth of a baby (Marks et al., 1992). Rates of depression among men in the postpartum period appear to be low: $3 \%$ of fathers in the Avon Longitudinal Study of Pregnancy and Childhood (Deater-Deckard et al., 1998) and 2.8\% of Australian fathers 4 months postpartum. (Matthey et al., 2000). Matthey and colleagues (2000) suggest that this may be due to under reporting of distress by men and that screening instruments may require different cut off scores to detect clinically significant symptomatology in men than in women.

There is ample evidence that the experience of criticism, coercion, control, humiliation or verbal or physical violence in an intimate relationship perpetrated by a partner on whom an individual is dependent is causally linked to the development of depression and anxiety in women. Although men are more likely to externalise and act out, rather than internalise their distress the assessment of intimate partner coercion, intimidation and violence is strikingly absent in most research on the etiology of postpartum depression. Studies (Boyce et al., 1991; Matthey et al., 2000) using the Intimate Bonds Measure (Wilhelm \& Parker, 1988) found that women whose partnerships were distinguished by high levels of control and low levels of care were at increased risk of developing postpartum depression, but fear of intimidation or actual experience of abuse were not ascertained.

There is very little research available on the psychological functioning of fathers of multiple infants, but consistent evidence that the quality of marital relationship is of central importance to maternal mental health after multiple births. Hay et al. (1990) reported that $37.5 \%$ of the studied parents reported that the birth of twins had imposed "great stress" on their relationship. These investigators concluded that "the one thing that characterises mothers who cope well ... after the arrival of twins is that they have a good secure relationship with a partner". The quality of marital relationship can be compromised in those who are still dealing with the psychological sequelae of infertility and assisted reproduction (Klock, 2001). Father's preparedness to embrace a multiple pregnancy and make voluntary adjustment in terms of relinquishment of independent professional and leisure activities in service of increased involvement in infant care and unpaid household work is of central importance. It appears that the birth of multiple infants may elicit greater paternal involvement than the birth of a single baby. Akerman (1997) in a study of parents of triplets found that fathers were closely and competently involved in infant care and felt emotionally close to their babies. Fathers who are closely involved in daily infant care from birth report greater enjoyment and satisfaction than those who are not (Weigel et al., 2000). This involvement may not be sustained. Garel and Blondel (1992) found that $50 \%$ of fathers of triplets had stopped providing care for their infants 1-year postpartum and that all the marital relationships were strained.

Social support, including absence of attachment to a peer group, few confiding relationships and lack of assistance in crises has been found to be related to postpartum depression. General dissatisfaction with support, rather than specific characteristics of number or quality of relationships, appears to be relevant (Scottish Intercollegiate Guidelines Network, 2002). It is common for parents of multiple infants to report social isolation, which appears to be the product both of practical difficulties in participating in social activities external to home and that parents of single infants may lack awareness of or empathy for the realities of mothering multiple infants. It is much more difficult to arrange childcare for multiple infants and members of the extended family may be less likely to offer to care for multiple infants at once.

There is a consistent finding that the financial consequences of having multiple infants can impose a major psychological burden. Many will already have incurred the substantial financial costs of infertility treatment. Complicated childbirth may incur costs of prolonged hospitalization for mother and or infants. Practical realities including having to purchase a larger car, housing of insufficient size, high medical costs, multiplied costs of infant furniture and equipment are associated with elevated anxiety (Hay et al., 1990; Klock, 2001). Few publicly funded forms of household help are available and families with multiple infants may have fewer financial resources to spend on services of this kind. These expenses usually coincide with the reduction in family income associated with women having to leave the paid workforce, many of whom will not have access to paid maternity leave.

Childbirth occurs in a life context, and there is consistent evidence that if women are having to attend to coincidental adverse life events such as bereavement, serious illness in a family member, conflict with friends or serious financial predicament at the same time as caring for an infant, psychological adjustment to parenthood is more difficult and distressing (Scottish Intercollegiate Guidelines Network, 2002). The most common of these for parents of multiple infants is perinatal bereavement. Loss of an infant either in pregnancy at birth or in the neonatal period is more likely in multiple than in single pregnancies. Stillbirth or neonatal death of one of a multiple precipitates the 
complex coincidence of death and detachment from one infant with the formation of attachment to another. Prematurity or infant disability also involves grief for the loss of an imagined child and the gradual adjustment to the real infant (Klock, 2001; Weigel et al., 2000). Thorpe et al. (1991) found that the most distressed mothers of twin 5-year-olds were those with a prolonged and inexpressible sadness associated with losing one twin when the other was living. Complicated grief is associated with higher rates of depression.

There is growing evidence that infant factors can exert an independent effect on maternal mood and the growth of a confident maternal identity (Murray et al., 1996). Mothers of multiple infants consistently report that they cannot meet the competing needs of more than one highly dependent infant, that feeding and settling are more problematic and that there is a constant sense of guilt about having to ignore one child while caring for the other (Garel \& Blondel, 1992). Premature or disabled infants are more fragile, require more vigilant care and are more difficult to feed (Bryan \& Denton, 2001). Caring for an infant with a difficult temperament or unsettled behaviour contributes to maternal exhaustion and a sense of powerlessness and ineffectiveness in mothers of newborns (Fisher et al., 2002). Hay and colleagues (1990) reported that twins were significantly more likely than single children to have the difficult temperamental features of high reactivity, low adaptability and more negative mood. There are reports that in these circumstances parental irritability and anger may be less contained and higher rates of child abuse have been reported among families with twins than with single children (Leonard, 1998).

There is increasing evidence that poor physical health after childbirth contributes to poor mental health (Brown, 1998). Physical problems related to birth can persist for months and are often undiagnosed and untreated. Mothers of multiple infants are much more likely to have experienced operative childbirth which is associated with prolonged physical recovery. Not breastfeeding has been associated with increased likelihood of postpartum depression and has been suggested to be an effective early indicator of vulnerability (Eberhard-Gran et al., 2002). The establishment of breastfeeding is more difficult for mothers of multiples. The effects of length of stay in hospital after childbirth on physical and psychological recovery are equivocal. Profound fatigue is widespread among mothers of newborns (Brown, 1998), but is often normalised or trivialised, despite the adverse impact it exerts on normal daily functioning. All mothers of multiple infants report severe fatigue and an excessive workload (Klock, 2001). Fatigue has been regarded as symptomatic of depression, but an alternative view is that it arises because the unpaid workload of mothering newborns is severely underestimated and not dignified with the awareness of occupational health and safety that apply in places of employment. Vital exhaustion may be a precursor for the development of depression in women whose unpaid workloads are neither acknowledged nor shared (Fisher et al., 2002). Large community surveys have not found an effect of early discharge on maternal mood (Brown, 1998), but $20 \%$ of women admitted to a residential service for treatment of early parenting difficulties reported that their maternity stay had been too short (Fisher et al., 2002).

Previous personal history of mood disorder, past psychiatric hospitalization and anxious or depressed mood in pregnancy are consistently found to predict postpartum depression (Scottish Intercollegiate Guidelines Network, 2002). Elevated family histories of psychiatric illness, in particular mood disorder and alcoholism, are reported among women with postpartum depression. Low parental care and neglect in childhood (Boyce et al., 1998) and childhood sexual and physical abuse (Buist \& Janson, 2001) contribute to adult depression and appear to be associated with postpartum mood disorders. The interaction between personal or family history of psychiatric illness, multiple births and postpartum depression has not been investigated to date.

Overall, while there is a growing body of evidence regarding the links between perinatal mental health and multiple births, there are substantial limitations in the existing literature. There is some evidence regarding the contribution of mothering multiple infants to some risk factors for postpartum depression and anxiety, which probably explains the elevated rates of these conditions in this population. However, much less is known about the relationships between multiple gestations and pregnancy mental health and between multiple births and severe perinatal mental illness. The links between psychiatric history, exposure to childhood abuse, intimate partner intimidation and depression after multiple births are unknown. There is only limited evidence about whether there are psychological differences between multiple births after spontaneous conception or assisted conception. There is a need for much more systematic comparative investigation of the effects of multiple gestations and births on the psychological functioning of fathers.

\section{Implications for Practice}

Although there are no randomised controlled trials of psychosocial interventions to prevent or treat perinatal mental illness in parents of multiple infants, there is sufficient existing knowledge to suggest some considerations for clinical practice.

The risks associated with multiple gestations, including pregnancy loss, operative childbirth, prematurity and infant morbidity should be discussed realistically with people seeking fertility treatment, who are at risk of idealising the notion of an instant family. Discussions should include the realities of parenting multiples and not be confined to pregnancy and the intrapartum period (Hay et al., 1990; Weigel et al., 2000). A non-judgmental empathic response from professional staff at time of diagnosis of multiple pregnancy, which does not presume that it will be greeted with joy, is vital.

Health care professionals should encourage honest exploration of the difficulties associated with multiple births, including the potential impact on way of life, marital relationship, financial security and body image and convey the notion that ambivalence is normal. Honest ventilation of anxieties, fears of being unprepared and 
overwhelmed and possible regrets about assisted conception is useful. Active planning for management of the workload (which is usually underestimated) and conflicts associated with parenting multiples should be encouraged in pregnancy (Hay et al., 1990; Klock, 2001). This should include plans to maximise assistance with childcare, partner availability, support from family of origin and suitable housing and transport. Advice regarding bed rest in advanced pregnancy needs to take into account the practicalities of employment and income generation and the competing needs of older children (Hay et al., 1990). All authors concur about the importance of including fathers specifically in these processes from the beginning of pregnancy care.

It is also relevant to assess in pregnancy, risk factors for postpartum depression apart from multiple gestation. These include personal or family history of psychiatric illness or childhood abuse; current depression; presence of marital difficulties, especially an inability to confide or fear of intimidation; fixed gender stereotypes regarding household work and infant care; lack of availability of practical support from family of origin or paid employees; limited network of social relationships and economic difficulties likely to arise as the result of multiple births.

If operative childbirth is planned, women need to be informed about the procedures to be used and to be permitted as much choice as possible (Fisher et al., 1997). If the infants are likely to require admission to neonatal intensive care, it is helpful for women to meet the neonatologist and tour the NICU prior to giving birth. Secure emotional attachment is fostered by minimizing separation of mother and baby and by assisting parents to provide infant care from birth. If depression after childbirth in a mother of multiple infants is recognised clinicians in this field need to appreciate the particular potential for multiple gestations and births to be associated coincidentally with bereavement and unrecognised loss. Grief can be complicated when it coincides with birth and its expression can be prohibited unless these competing dual processes are acknowledged.

If it is suspected that a mother of multiple infants is depressed, early referral to a specialist service and active management are indicated. The risks and benefits of antidepressant pharmacotherapy need to be discussed with particular consideration of their implications for breastfeeding. Assessment of whether the mother's distress poses a risk to her infant should take into account mother-infant interaction, whether she has age appropriate expectations of infant behaviour and appropriate attribution of infant motives. It is important to provide active encouragement of additional practical and emotional support from her partner, family of origin and professional services and not to suggest that use of these implies less maternal efficacy. Regular scheduled periods of relief from infant care and unpaid household work are restorative of maternal wellbeing. If a woman is severely unwell, then admission to a specialist early-parenting or psychiatric mother-baby service is indicated. There is emerging evidence that if the infant's unsettled behaviour is effectively treated, and a regular feeding and sleeping schedule is established, maternal mood improves (Armstrong et al., 2000). However, the particular benefits of this approach to parents of twins or higher multiples have not yet been shown.

Although the birth of multiple infants carries additional risks to psychological wellbeing compared to the birth of a single infant, professional care which offers encouragement and conveys optimism about the capacity of parents to adapt to these demands is of great value.

\section{Acknowledgments}

The authors are very grateful to Ms Karin Hammarberg for comments on earlier drafts of this paper.

\section{References}

Akerman, B. A., Hovmoller, M., \& Thomassen, P. A. (1997). The challenges of expecting, delivering and rearing triplets. Acta Geneticae Medicae et Gemellologiae, 46, 81-86.

Armstrong, K. L., Fraser, J. A., Dadds, M. R., \& Morris, J. (2000). Promoting secure attachment, maternal mood and child health in a vulnerable population: A randomised controlled trial. Journal of Paediatrics and Child Health, 36, 555-562.

Barnett, B., Lochart, K., Bernard, D., Manicavasagar, V., \& Dudley, M. (1993). Mood disorders among mothers of infants admitted to a mothercraft hospital. Journal of Paediatrics and Child Health, 29, 270-275.

Barrett, J. F., \& Ritchie, W. K. (2002). Twin delivery. Best Practice and Research in Clinical Obstetrics and Gynaecology, 16(1), 43-56.

Boyce, P., Harris, M. B., Silove, D., Morgan, A., Wilhelm, K., \& Hadzi-Pavlovic, D. (1998). Psychosocial factors associated with depression: A study of socially disadvantaged women with young children. The Journal of Nervous and Mental Diseases, 186(1), 3-11.

Boyce, P., Hickie, I., \& Parker, G. (1991). Parents, partners or personality? Risk factors for post-natal depression. Journal of Affective Disorders, 21, 245-255.

Brown, S. (1998). Maternal health after childbirth: Results of an Australian population based survey. British Journal of Obstetrics and Gynaecology, 105, 156-161.

Bryan, E. (2002). Loss in higher multiple pregnancy and multifetal pregnancy reduction. Twin Research, 5(3), 169-174.

Bryan, E., \& Denton, J. (2001). Education for parents. In I. Blickstein, \& L. G. Keith (Eds.), Iatrogenic multiple pregnancy: Clinical implications (pp. 211-222). New York: The Parthenon Publishing Group.

Buist, A., \& Janson, H. (2001). Childhood sexual abuse, parenting and postpartum depression - A 3-year follow-up study. Child Abuse and Neglect, 25, 909-921.

Collopy, K. S. (2002). We didn't deserve this: Bereavement associated with multifetal reduction. Twin Research, 5(3), 231-235.

Colonna, F., Cuttini, M., Melon, F., \& de Vonderweid, U. (1997). The success of maternal feeding with very low birth weight premature infants, singletons and twins: A 10-year experience. Pediatricae Medicae Chirugica, 19(3), 159-163.

Colpin, H., De Munter, A., Nys, K., \& Vandenmeulebroecke, L. (1998). Prenatal attachment in future parents of twins. Early Development and Parenting, 7, 223-227. 
Condon, J., \& Watson, T. (1987). The maternity blues: Exploration of a psychological hypothesis. Acta Psychiatrica Scandinavia, 76, 164-171.

Deater-Deckard, K., Pickering, K., Dunn, J., \& Golding, J. (1998). Family structure and depressive symptoms in men preceding and following the birth of a child. The American Journal of Psychiatry, 155(6), 818-823.

Eberhard-Gran, M., Eskild, A., Tambs, K., Samuelsen, S. O., \& Opjordsmoen, S. (2002). Depression in postpartum and non-postpartum women: Prevalence and risk factors. Acta Psychiatrica Scandiniavica, 106, 426-433.

Epperson, N. (1999). Postpartum major depression: Detection and treatment. American Family Physician, 59(8), 2247-2254.

Evans, J., Heron, J., Francomb, H., Oke, S., \& Golding, J. (2001). Cohort study of depressed mood during pregnancy and after childbirth. British Medical Journal, 323(7307), 257-260.

Fisher, J., Astbury, J., \& Smith, A. (1997). Adverse psychological impact of operative obstetric interventions: A prospective study. Australian and New Zealand Journal of Psychiatry, 31, $728-738$.

Fisher, J. R. W. (2002). Difficult deliveries disenfranchised grief and delayed trauma reactions. In M. Kirkman, J. M. Maher, \& K. Torney Souter (Eds.), The fertile imagination: Narratives of reproduction. Meridian, The La Trobe University English Review (Vol. 18, pp. 39-58). Melbourne: Meridian.

Fisher, J. R. W., Feekery, C. J., \& Rowe-Murray, H. J. (2002). Nature, severity and correlates of psychological distress in women admitted to a private mother-baby unit. Journal of Paediatrics and Child Health, 38, 140-145.

Garel, M., \& Blondel, B. (1992). Assessment at 1 year of the psychological consequences of having triplets. Human Reproduction, 7(5), 729-732.

Gleicher, N., Campbell, D. P., Chan, C. L., Karande, V., Rao, R., Balin, M., et al. (1995). The desire for multiple birth in couples with infertility problems contradicts present practice patterns. Human Reproduction, 10(5), 1079-1084.

Goldfarb, J. D., Kinzer, D. J., Boyle, M., \& Kurit, D. (1996). Attitudes of in vitro fertilization and intrauterine insemination couples toward multiple gestation pregnancy and multifetal pregnancy reduction. Fertility and Sterility, 65(4), $815-820$.

Hay, D. A., Gleeson, C., Davies, C., Lorden, B., Mitchell, D., \& Paton, L. (1990). What information should the multiple birth family receive before, during and after the birth? Acta Geneticae Medicae et Gemellologiae, 39, 259-269.

Johnstone, S., Boyce, P., Hickey, A., Morris-Yates, A., \& Harris, M. (2001). Obstetric risk factors for postnatal depression in urban and rural community samples. Australian \& New Zealand Journal of Psychiatry, 35(1), 69-74.

Kendell, R., McGuire, R., Connor, Y., \& Cox, J. (1981). Mood changes in the first three weeks following childbirth. Journal of Affective Disorders, 3(4), 317-326.

Klock, S. C. (2001). The transition to parenthood. In I. Blickstein \& L. G. Keith (Eds.), Iatrogenic multiple pregnancy: Clinical implications (pp. 225-234). New York: The Parthenon Publishing Group.

Kollantai, J., \& Fleischer, L. M. (2001). Insert 16.3 realities: Coping with the impact of death in a multiple pregnancy. In I. Blickstein \& L. G. Keith (Eds.), Iatrogenic multiple pregnancy: Clinical implications (pp. 216-217). New York: The Parthenon Publishing Group.

Leiblum, S. R., Kemmann, E., \& Taska, L. (1990). Attitudes toward multiple birth and pregnancy concerns in infertile and non-infertile women. Journal of Psychosomatic Obstetrics and Gynaecology, 11, 197-210.

Leonard, L. G. (1998). Depression and anxiety disorders during multiple pregnancy and parenthood. Journal of Obstetric, Gynecologic, and Neonatal Nursing, 27(3), 329-377.

Loo, K. K., Espinosa, M., Tyler, R., \& Howard, J. (2003). Using knowledge to cope with stress in the NICU: How parents integrate learning to read the physiologic and behavioral cues of the infant. Neonatal Networks, 22(1), 31-37.

Marks, M. N., Wieck, A., Checkley, S. A., \& Kumar, R. (1992). Contribution of psychological and social factors to psychotic and non-psychotic relapse after childbirth in women with previous histories of affective disorder. Journal of Affective Disorders, 29, 253-264.

Matthey, S., Barnett, B., Ungerer, J., \& Waters, B. (2000). Paternal and maternal depressed mood during the transition to parenthood. Journal of Affective Disorders, 60, 75-85.

Murray, L., Sinclair, D., Cooper, P. J., Ducournau, P., \& Turner, P. (1999). The socioemotional development of 5-year-old children of postnatally depressed mothers. Journal of Child Psychology and Psychiatry, 40(8), 1259-1271.

Murray, L., Stanley, C., Hooper, R., King, F., \& Fiori-Cowley, A. (1996). The role of infant factors in postnatal depression and mother-infant interactions. Developmental Medicine and Child Neurology, 38, 109-119.

O'Hara, M. W., Schlechte, J. A., Lewis, D. A., \& Varner, M. W. (1991). Controlled prospective study of postpartum mood disorders: Psychological, environmental, and hormonal variables. Journal of Abnormal Psychology, 100(1), 63-73.

Pector, E. A., \& Smith-Levitin, M. (2002). Mourning and psychological issues in multiple birth loss. Seminars in Neonatology, 7(3), 247-256.

Pfuhlmann, B., Stoeber, G., \& Beckmann, H. (2002). Postpartum psychoses: Prognosis, risk factors, and treatment. Current Psychiatry Reports, 4, 185-190.

Robin, M., Bydlowski, M., Cahen, F., \& Josse, D. (1991). Maternal reactions to the birth of triplets. Acta Geneticae Medicae et Gemellologiae, 40, 41-51.

Rowe-Murray, H., \& Fisher, J. (2002). Baby friendly hospital practices: Cesarean section is a persistent barrier to early initiation of breastfeeding. Birth, 29(2), 124-130.

Scottish Intercollegiate Guidelines Network (2002). Postnatal depression and puerperal psychosis. A national clinical guideline. Edinburgh: Royal College of Physicians.

Steiner, M. (1998). Further needs in clinical assessment Perinatal mood disorders: Position paper. Psychopharmacology Bulletin, 34(3), 301-307.

Swanson, P. B., Pearsall-Jones, J. G., \& Hay, D. A. (2002). How mothers cope with the death of a twin or higher multiple. Twin Research, 5(3), 156-164.

Thorpe, K., Golding, J., MacGillivray, I., \& Greenwood, R. (1991). Comparison of prevalence of depression in mothers of twins and mothers of singletons. British Medical Journal, 302, 875-878. 
Weigel, D. J., Auxier, C. R., \& Frye, T. M. (2000). Multiplebirthing trends: Impact on couples and families. Family Journal, 8(4), 380-386.
Wilson, L. M., Reid, A. J., Midmer, D.K., Biringer, A., Carroll, J.C., \& Stewart, D. E. (1996). Antenatal psychosocial risk factors associated with adverse postpartum family outcomes. Canadian Medical Association Journal, 154(6), 785-799.

\section{Helping your patients become parents...}

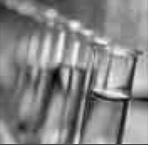

Sydney IVF, one of the world's leading fertility clinics, is proud to support the International Society for Twin Studies.

We can treat infertile couples with a wide range of options including:

- Assisted insemination
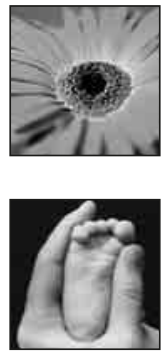

- IVF (ICSI if needed)

- Preimplantation genetic diagnosis (PGD)

- Surgical sperm collection

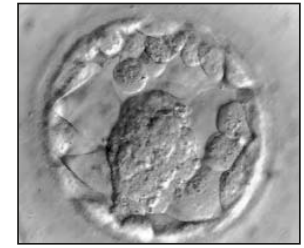
- ESA and TESE

- Cryogenic storage of gametes and embryos

- Investigation and treatment of recurrent miscarriage

- Prenatal testing for genetic disease

\section{Single Embryo Transfer}

By leading in blastocyst culture and blastocyst cryostorage, Sydney IVF is the first clinic in the world to demonstrate that the chance of a baby is now as high or higher when only one fresh embryo is transferred instead of the more usual two, while greatly reducing the risk of twins, with its attendant greater neonatal morbidity and mortality.

\section{Referrals}

A complete list of Sydney IVF's accredited doctors and their contact details is available at www.sivf.com.au or phone (02) 92215964

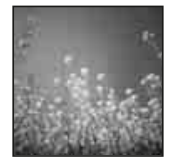

Clinics at: Sydney, Liverpool, Canberra, Wollongong, Newcastle, Orange, Lismore, Coffs Harbour, Tamworth and Launceston

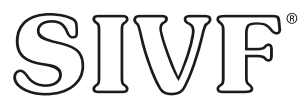

Sydney IVF 